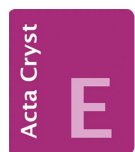

CRYSTALLOGRAPHIC COMMUNICATIONS

ISSN 2056-9890

Received 4 January 2016

Accepted 1 February 2016

Edited by M. Weil, Vienna University of

Technology, Austria

Keywords: crystal structure; garnet; lithium lanthanum zirconate (LLZO); symmetry reduction

CCDC reference: 1451178

Supporting information: this article has supporting information at journals.iucr.org/e

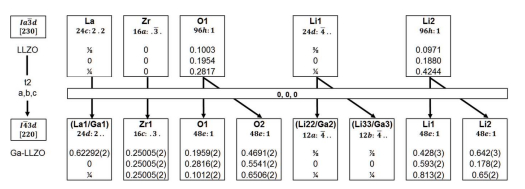

OPEN $\odot$ ACCESS

\section{Symmetry reduction due to gallium substitution in the garnet $\mathrm{Li}_{6.43(2)} \mathrm{Ga}_{0.52(3)} \mathrm{La}_{2.67(4)} \mathrm{Zr}_{2} \mathrm{O}_{12}$}

\author{
Lars Robben, ${ }^{\mathrm{a} *}$ Elena Merzlyakova, ${ }^{\mathrm{b}}$ Paul Heitjans ${ }^{\mathrm{b}}$ and Thorsten M. Gesing ${ }^{\mathrm{a}}$ \\ ${ }^{a}$ Chemische Kristallographie fester Stoffe, Institut für Anorganische Chemie und Kristallographie, FB02, Leobener Strasse/ \\ NW2, and MAPEX Center for Materials and Processes, Universität Bremen, Bibliotheksstrasse 1, 28359 Bremen, \\ Germany, and ${ }^{\mathbf{b}}$ Institut für Physikalische Chemie und Elektrochemie, Leibniz Universität Hannover, Callinstrasse 3-3a, \\ D-30167 Hannover, Germany. ${ }^{*}$ Correspondence e-mail: Irobben@uni-bremen.de
}

Single-crystal structure refinements on lithium lanthanum zirconate (LLZO; $\mathrm{Li}_{7} \mathrm{La}_{3} \mathrm{Zr}_{2} \mathrm{O}_{12}$ ) substituted with gallium were successfully carried out in the cubic symmetry space group $I \overline{4} 3 \mathrm{~d}$. Gallium was found on two lithium sites as well as on the lanthanum position. Due to the structural distortion of the resulting $\mathrm{Li}_{6.43(2)} \mathrm{Ga}_{0.52(3)} \mathrm{La}_{2.67(4)} \mathrm{Zr}_{2} \mathrm{O}_{12}$ (Ga-LLZO) single crystals, a reduction of the LLZO cubic garnet symmetry from $I \mathrm{a} \overline{3} d$ to $I \overline{4} 3 d$ was necessary, which could hardly be analysed from X-ray powder diffraction data.

\section{Chemical context}

Garnets can be described with the ideal formula $A_{3} B_{2}\left(X_{\mathrm{O}_{4}}\right)_{3}$ in space group $I \mathrm{a} \overline{3} d$, with different coordination polyhedra of the respective elements with oxygen, resulting in a distorted cube for $A$ (e.g. Ca), an octahedron for $B($ e.g. Al) and a tetrahedron for $X(e . g$. Si). The variability of the elements on the crystallographic sites (thereby keeping the high symmetry) gives rise to interesting material properties like ferrimagnetism (Geller, 1967). In recent years, garnet-type compounds containing Li have gained considerable interest as promising electrolyte materials for all-solid-state Li-ion batteries. The so-called 'Li-stuffed' garnets, which contain more $\mathrm{Li}$ than available on tetrahedral sites $(X)$, meaning that excess $\mathrm{Li}$ occupies other sites as well, show an increase in $\mathrm{Li}$ ion mobility. An exhaustive overview of these compounds was recently given by Thangadurai et al. (2014). The garnet-type fast lithium ion conductor $\mathrm{Li}_{7} \mathrm{La}_{3} \mathrm{Zr}_{2} \mathrm{O}_{12}$, abbreviated as LLZO, is such an 'Li-stuffed' garnet. Awaka et al. (2009) described the crystal structure of pure LLZO at ambient conditions in space group $I_{1} / a c d$. Even a small amount of $\mathrm{Al}$ in the structure (Al-LLZO) stabilizes the cubic garnet symmetry described in space group $I \mathrm{a} \overline{3} d$ by Geiger et al. (2011). These authors reported that Al could be found on two different tetrahedral sites using ${ }^{27} \mathrm{Al}$ MAS NMR spectroscopy but a final analysis was not possible due to the minor $\mathrm{Al}$ content. Rettenwander et al. (2014) reported on ${ }^{71} \mathrm{Ga}$ MAS NMR spectroscopy measurements on gallium substituted $\mathrm{Li}_{7}$. ${ }_{3 x} \mathrm{Ga}_{x} \mathrm{La}_{3} \mathrm{Zr}_{2} \mathrm{O}_{12}$ (Ga-LLZO) indicating a fourfold coordination of the gallium atoms. The authors excluded the presence of $\mathrm{Ga}$ at the $24 d$ position $(I \mathrm{a} \overline{3} d)$ and assumed that the local symmetry could be lower than indicated by diffraction methods. In principle, the following exchanges are possible: $(i)$ $3 \mathrm{Li}^{+} \leftrightarrow \mathrm{Ga}^{3+}+2$ voids, which is the most probable one and yields a good explanation for the higher conductivity due to the higher lithium atom jump probability to empty positions as 


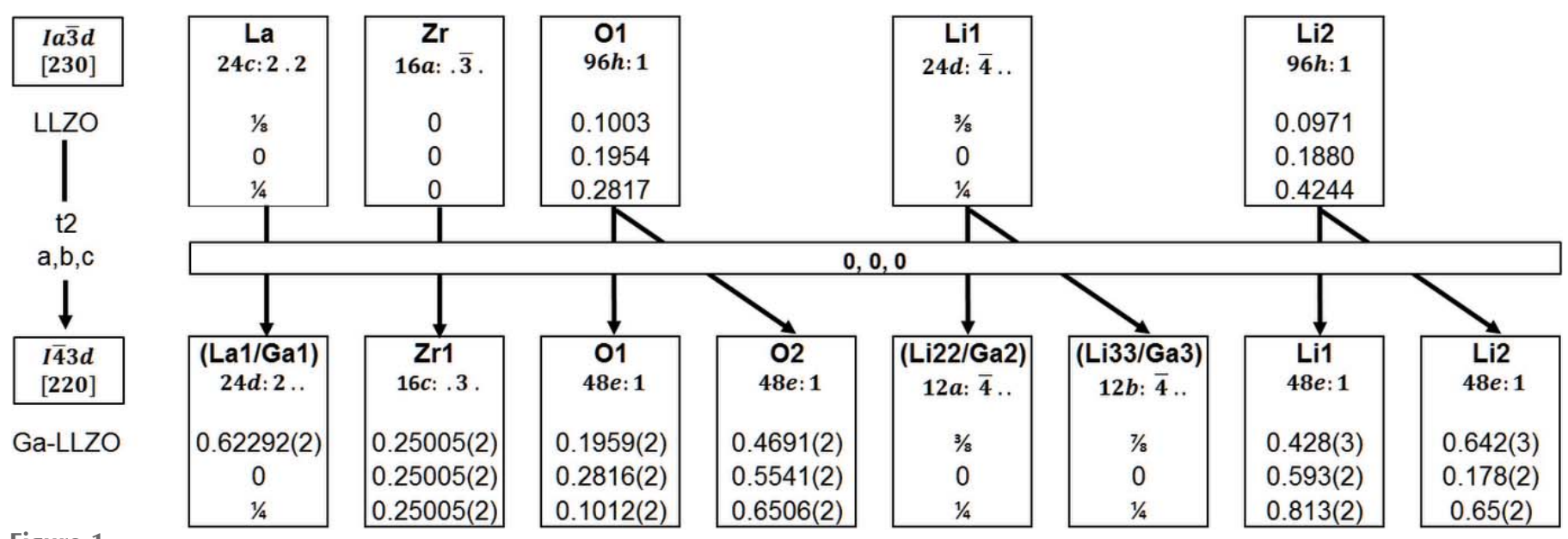

Figure 1

Bärnighausen tree (Bärnighausen, 1980) of the group-subgroup relation between cubic LLZO and the symmetry-reduced cubic Ga-LLZO.

discussed (Rettenwander et al., 2014); (ii) $\mathrm{La}^{3+} \leftrightarrow \mathrm{Ga}^{3+}$, a valence-neutral exchange which should lead to a dynamical
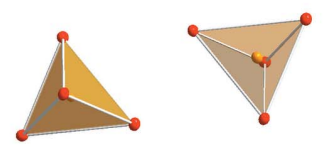

$\mathrm{Li} 22 / \mathrm{Ga} 2$
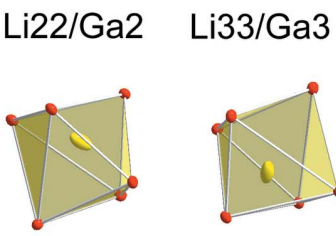

Li1

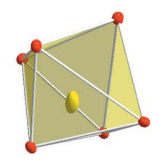

Li2

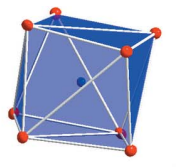

La1/Ga1

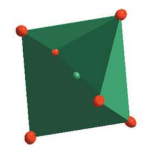

$\mathrm{Zr} 1$
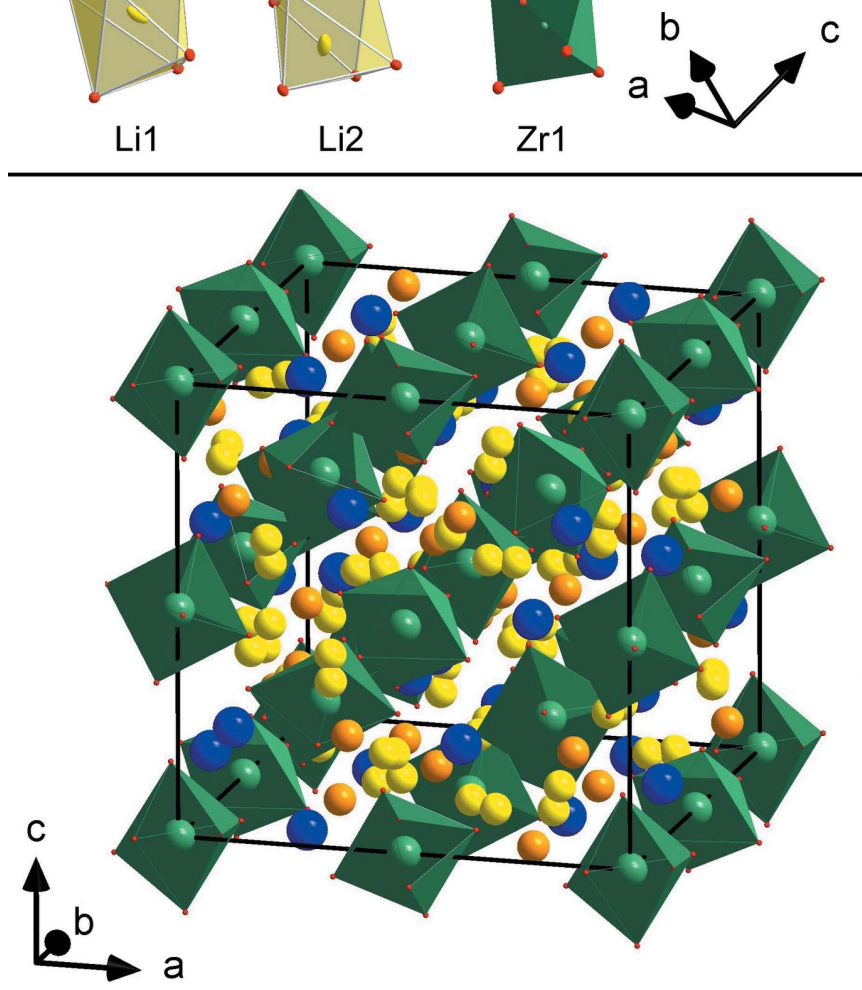

Figure 2

Crystal structure of $\mathrm{Li}_{6.43(2)} \mathrm{Ga}_{0.52(3)} \mathrm{La}_{2.67(4)} \mathrm{Zr}_{2} \mathrm{O}_{12}$ (Ga-LLZO) with all possible atom positions between the $\mathrm{ZrO}_{6}$ octahedra (bottom) and the atom position specific coordination polyhedra (top). Displacement ellipsoids (top) are given at the $50 \%$ probability level. disorder of the gallium atoms in order to lower the coordination number and shorten the $\mathrm{Ga}-\mathrm{O}$ bond lengths for bondvalence balance, taking the different radii into account. The valence-neutral exchange should finally lead to higher displacement parameters of the atoms on the lanthanum position compared to that of the lighter zirconium atoms. (iii) $\mathrm{Zr}^{4+} \leftrightarrow \mathrm{Ga}^{3+}+\mathrm{Li}^{+}$, which needs slightly more lithium for charge balance and could therefore be of minor probability.

\section{Structural commentary}

The unit cell of the obtained single crystals could be well indexed using a body-centered cubic lattice with lattice parameter $a=12.9681$ (15) ̊. The space group determination with XPREP (Bruker, 2014) leads at once to the highest possible space group $I \mathrm{a} \overline{3} d$. However, a satisfactory structure solution or refinement with published structural data (Geiger et al., 2011) in this space group type was not possible. Consequently, structure solutions by charge flipping (Bruker, 2009) were tried in all possible subgroups of $I \mathrm{a} \overline{3} d$ and the lowest $R$-values were obtained for the charge-flipping run in space group $I \overline{4} 3 \mathrm{~d}$. Subsequent refinements lead to the present structure model and clearly indicate the substitution of $\mathrm{Ga}^{3+}$ on the former $24 c$ $\mathrm{La}^{3+}$ site as well as $24 d \mathrm{Li}^{+}$site in the aristotype in space group $I \mathrm{a} \overline{3} d$. The latter site splits into two sites due to the symmetry reduction as indicated by the Bärnighausen tree (Bärnighausen, 1980) given in Fig. 1. The deviation from six symmetry-equivalent $\mathrm{Zr}-\mathrm{O}$ distances in LLZO $(\mathrm{Ia} \overline{3} d)$ results in a distortion of the $\mathrm{ZrO}_{6}$ octahedron with $\mathrm{Zr}-\mathrm{O}$ distances of $3 \times 2.095$ (2) and $3 \times 2.113(2) \AA$ in Ga-LLZO. Another significant reduction of the highest possible symmetry for LLZO is the distortion of the eightfold coordinate La position (Fig. 2), for which distances between 2.496 (2) and 2.595 (2) $\AA$ are found in Ga-LLZO. This distortion results from the splitting of the $96 h$ position of the oxygen atom in $I \mathrm{a} \overline{3} d$ into two $48 e$ positions in $I \overline{4} 3 d$ (Fig. 1). Because the two lithium positions ( $\mathrm{Li} 22$ and Li32) occupied by gallium are in principle identical to those positions of the higher symmetry structure (but with slightly shorter bond length due to the gallium 
Table 1

Experimental details.

\begin{tabular}{|c|c|}
\hline \multicolumn{2}{|l|}{ Crystal data } \\
\hline Chemical formula & $\mathrm{Li}_{6.43} \mathrm{Ga}_{0.52} \mathrm{La}_{2.67} \mathrm{Zr}_{2} \mathrm{O}_{12}$ \\
\hline$M_{\mathrm{r}}$ & 826.20 \\
\hline Crystal system, space group & Cubic, $I \overline{4} 3 d$ \\
\hline Temperature $(\mathrm{K})$ & 301 \\
\hline$a(\AA)$ & $12.9681(15)$ \\
\hline$V\left(\AA^{3}\right)$ & $2180.9(8)$ \\
\hline$Z$ & 8 \\
\hline Radiation type & Мо $K \alpha$ \\
\hline$\mu\left(\mathrm{mm}^{-1}\right)$ & 13.41 \\
\hline Crystal size $(\mathrm{mm})$ & $0.25 \times 0.15 \times 0.13$ \\
\hline \multicolumn{2}{|l|}{ Data collection } \\
\hline Diffractometer & Bruker APEXII CCD \\
\hline Absorption correction & $\begin{array}{l}\text { Multi-scan (SADABS; Bruker, } \\
\text { 2014) }\end{array}$ \\
\hline$T_{\min }, T_{\max }$ & $0.495,0.754$ \\
\hline $\begin{array}{l}\text { No. of measured, independent and } \\
\text { observed }[I>2 \sigma(I)] \text { reflections }\end{array}$ & $472450,3678,3508$ \\
\hline$R_{\text {int }}$ & 0.046 \\
\hline$(\sin \theta / \lambda)_{\max }\left(\AA^{-1}\right)$ & 1.340 \\
\hline \multicolumn{2}{|l|}{ Refinement } \\
\hline$R\left[F^{2}>2 \sigma\left(F^{2}\right)\right], w R\left(F^{2}\right), S$ & $0.026,0.056,1.46$ \\
\hline No. of reflections & 3678 \\
\hline No. of parameters & 50 \\
\hline No. of restraints & 3 \\
\hline$\Delta \rho_{\max }, \Delta \rho_{\min }\left(\mathrm{e} \AA^{-3}\right)$ & $2.08,-1.91$ \\
\hline Absolute structure & $\begin{array}{l}\text { Flack } x \text { determined using } 1493 \\
\quad \text { quotients }\left[\left(I^{+}\right)-\left(I^{-}\right)\right] /\left[\left(I^{+}\right)+\left(I^{-}\right)\right] \\
\quad \text { (Parsons et al., 2013) }\end{array}$ \\
\hline Absolute structure parameter & $0.045(9)$ \\
\hline
\end{tabular}

Computer programs: APEX2 and SAINT (Bruker, 2014), TOPAS (Bruker, 2009), SHELXL2014 (Sheldrick, 2015), DIAMOND (Brandenburg, 1999) and publCIF (Westrip, 2010).

substitution, viz. 4 × 1.916 (1) $\AA$ in LLZO and 4 × 1.908 (2) $\AA$ in Ga-LLZO), and the Li1 and Li2 positions are not occupied by gallium, the symmetry reduction is a confirmation of gallium atoms to be found also on the lanthanum position. This is also supported by the higher displacement parameter of the La site compared to the $\mathrm{Zr}$ site, as explained previously.

\section{Synthesis and crystallization}

The synthesis was configured to yield a compound with nominal composition $\mathrm{Li}_{6.25} \mathrm{Ga}_{0.25} \mathrm{La}_{3} \mathrm{Zr}_{2} \mathrm{O}_{12}$. $2 \mathrm{~g}$ of a stoichiometric mixture of the pre-dried ( $30 \mathrm{~h}$ at $373 \mathrm{~K}$ in vacuum) educts $\mathrm{Li}_{2} \mathrm{O}$ (with an excess of $10 \%$ wt to compensate the lithium loss due to thermal treatment), $\mathrm{La}_{2} \mathrm{O}_{3}, \mathrm{ZrO}_{2}$ and $\mathrm{Ga}_{2} \mathrm{O}_{3}$ was weighted into a WC milling beaker $(45 \mathrm{ml}, 100 \mathrm{WC}$ milling balls of $5 \mathrm{~mm}$ diameter, Fritsch, Germany) under inert conditions (glovebox) and high-energy ball-milled in a planetary ball mill (Pulverisette 7 premium line, Fritsch, Germany) under argon atmosphere for $8 \mathrm{~h}$ at a rotational speed of $10 \mathrm{~s}^{-1}$ as reported previously (Düvel et al., 2012) for Al-substituted LLZO. The obtained powder was pressed to a pellet using a uniaxial pressure of $0.8 \mathrm{GPa}$. A stack of three pellets was placed on a platinum ring seated on a corundum plate, covered with a corundum crucible and heated for $12 \mathrm{~h}$ at $1323 \mathrm{~K}$ in a muffle furnace before cooling to room-temperature. The middle pellet from the stack had smooth green color and showed visible grains. The surface of the pellet was grey and brittle and consisted mainly of lanthanum zirconates due to Li loss. From this pellet single crystals were extracted using a polarization microscope. Rietveld refinement of X-ray powder diffraction data of the green product shows a mixture of $96.8(9) \%_{\mathrm{wt}}$ cubic garnet-type Ga-LLZO and $3.2(9) \%_{\mathrm{wt}}$ $\mathrm{Li}_{2} \mathrm{ZrO}_{3}$ with a lattice parameter of $a=12.9738$ (19) $\AA$ for its garnet-type structure. Energy dispersive X-ray analysis of the single crystal gave a tentative formula of $\mathrm{Li}_{6.5(1)} \mathrm{Ga}_{0.5(1)^{-}}$ $\mathrm{La}_{2.8(1)} \mathrm{Zr}_{2.0(1)} \mathrm{O}_{12}$, in good agreement with the refined formula $\mathrm{Li}_{6.43(2)} \mathrm{Ga}_{0.52(3)} \mathrm{La}_{2.67(4)} \mathrm{Zr}_{2} \mathrm{O}_{12}$ determined from single crystal $\mathrm{X}$-ray diffraction data.

\section{Refinement}

Crystal data, data collection and structure refinement details are summarized in Table 1. Structure refinement was carried out as a two-component (merohedral) twin. Sites showing a statistical occupancy were constrained with respect to positions and anisotropic displacement parameters. An independent refinement of the anisotropic displacement parameters of $\mathrm{Ga}$ and $\mathrm{Li}$ on the $\mathrm{Ga} 2 / \mathrm{Li} 22$ and $\mathrm{Ga} 3 / \mathrm{Li} 33$ sites was not possible, although the reflection-to-parameter ratio is rather high. To ensure charge neutrality during the refinement of the $\mathrm{Ga}$ and $\mathrm{Li}$ occupancies on the Ga2/Li22 and Ga3/Li33 sites, the occupancies were restrained to exchange three $\mathrm{Li}$ atoms against one $\mathrm{Ga}$ atom.

\section{Acknowledgements}

Financial support of the Deutsche Forschungsgemeinschaft (DFG) in the Heisenbergprogram (TMG: GE1981/31, GE1981/32) and the Niedersächsisches Ministerium für Wissenschaft und Kultur (MWK) (PH: 74ZN994) is gratefully acknowledged.

\section{References}

Awaka, J., Kijima, N., Hayakawa, H. \& Akimoto, J. (2009). J. Solid State Chem. 182, 2046-2052.

Bärnighausen, H. (1980). Comm. Math. Comput. Chem. 9, 139-175.

Brandenburg, K. (1999). DIAMOND. Crystal Impact GbR, Bonn, Germany.

Bruker (2009). TOPAS. Bruker AXS Inc., Madison, Wisconsin, USA.

Bruker (2014). APEX2, SAINT, SADABS and XPREP. Bruker AXS Inc., Madison, Wisconsin, USA.

Düvel, A., Kuhn, A., Robben, L., Wilkening, M. \& Heitjans, P. (2012). J. Phys. Chem. C, 116, 15192-15202.

Geiger, C. A., Alekseev, E., Lazic, B., Fisch, M., Armbruster, T., Langner, R., Fechtelkord, M., Kim, N., Pettke, Th. \& Weppner, W. (2011). Inorg. Chem. 50, 1089-1097.

Geller, S. (1967). Z. Kristallogr. 125, 1-47.

Parsons, S., Flack, H. D. \& Wagner, T. (2013). Acta Cryst. B69, 249259.

Rettenwander, D., Geiger, C. A., Tribus, M., Tropper, P. \& Amthauer, G. (2014). Inorg. Chem. 53, 6264-6269.

Sheldrick, G. M. (2015). Acta Cryst. C71, 3-8.

Thangadurai, V., Narayanan, S. \& Pinzaru, D. (2014). Chem. Soc. Rev. 43, 4714-4727.

Westrip, S. P. (2010). J. Appl. Cryst. 43, 920-925. 


\section{supporting information}

Acta Cryst. (2016). E72, 287-289 [doi:10.1107/S2056989016001924]

Symmetry reduction due to gallium substitution in the garnet

$\mathrm{Li}_{6.43(2)} \mathrm{Ga}_{0.52(3)} \mathrm{La}_{2.67(4)} \mathrm{Zr}_{2} \mathrm{O}_{12}$

\section{Lars Robben, Elena Merzlyakova, Paul Heitjans and Thorsten M. Gesing}

Computing details

Data collection: APEX2 (Bruker, 2014); cell refinement: SAINT (Bruker, 2014); data reduction: SAINT (Bruker, 2014); program(s) used to solve structure: TOPAS (Bruker, 2009); program(s) used to refine structure: SHELXL2014 (Sheldrick, 2015); molecular graphics: DIAMOND (Brandenburg, 1999); software used to prepare material for publication: $p u b l C I F$ (Westrip, 2010).

Lithium gallium lanthanum zirconate

Crystal data

$\mathrm{Li}_{6.43} \mathrm{Ga}_{0.52} \mathrm{La}_{2.67} \mathrm{Zr}_{2} \mathrm{O}_{12}$

$M_{r}=826.20$

Cubic, $I \overline{4} 3 d$

$a=12.9681(15) \AA$

$V=2180.9(8) \AA^{3}$

$Z=8$

$F(000)=340$

$D_{\mathrm{x}}=5.033 \mathrm{Mg} \mathrm{m}^{-3}$

Data collection

Bruker APEXII CCD diffractometer

$\varphi$ and $\omega$ scans

Absorption correction: multi-scan

(SADABS; Bruker, 2014)

$T_{\min }=0.495, T_{\max }=0.754$

472450 measured reflections

\section{Refinement}

Refinement on $F^{2}$

Least-squares matrix: full

$R\left[F^{2}>2 \sigma\left(F^{2}\right)\right]=0.026$

$w R\left(F^{2}\right)=0.056$

$S=1.46$

3678 reflections

50 parameters

3 restraints

$w=1 /\left[\sigma^{2}\left(F_{\mathrm{o}}{ }^{2}\right)+(0.011 P)^{2}+8.6617 P\right]$

where $P=\left(F_{\mathrm{o}}^{2}+2 F_{\mathrm{c}}^{2}\right) / 3$
Mo $K \alpha$ radiation, $\lambda=0.71073 \AA$

Cell parameters from 9913 reflections

$\theta=3.1-72.3^{\circ}$

$\mu=13.41 \mathrm{~mm}^{-1}$

$T=301 \mathrm{~K}$

Irregular, green

$0.25 \times 0.15 \times 0.13 \mathrm{~mm}$

3678 independent reflections

3508 reflections with $I>2 \sigma(I)$

$R_{\text {int }}=0.046$

$\theta_{\text {max }}=72.3^{\circ}, \theta_{\text {min }}=2.2^{\circ}$

$h=-34 \rightarrow 34$

$k=-34 \rightarrow 34$

$l=-34 \rightarrow 34$

$(\Delta / \sigma)_{\max }=0.049$

$\Delta \rho_{\max }=2.08 \mathrm{e} \AA^{-3}$

$\Delta \rho_{\min }=-1.91$ e $\AA^{-3}$

Extinction correction: SHELXL2014 (Sheldrick, 2015), $\mathrm{Fc}^{*}=\mathrm{kFc}\left[1+0.001 \mathrm{xFc}^{2} \lambda^{3} / \sin (2 \theta)\right]^{-1 / 4}$

Absolute structure: Flack $x$ determined using 1493 quotients $\left[\left(I^{+}\right)-\left(I^{-}\right)\right] /\left[\left(I^{+}\right)+(I)\right]$ (Parsons et al., 2013)

Absolute structure parameter: 0.045 (9) 


\section{Special details}

Geometry. All e.s.d.'s (except the e.s.d. in the dihedral angle between two l.s. planes) are estimated using the full covariance matrix. The cell e.s.d.'s are taken into account individually in the estimation of e.s.d.'s in distances, angles and torsion angles; correlations between e.s.d.'s in cell parameters are only used when they are defined by crystal symmetry. An approximate (isotropic) treatment of cell e.s.d.'s is used for estimating e.s.d.'s involving 1.s. planes.

Refinement. Refined as a 2-component twin.

Fractional atomic coordinates and isotropic or equivalent isotropic displacement parameters $\left(\AA^{2}\right)$

\begin{tabular}{llllll}
\hline & $x$ & $y$ & $z$ & $U_{\text {iso }} * / U_{\text {eq }}$ & Occ. $(<1)$ \\
\hline La1 & $0.62292(2)$ & 0.0000 & 0.2500 & $0.00746(2)$ & $0.889(4)$ \\
Ga1 & $0.62292(2)$ & 0.0000 & 0.2500 & $0.00746(2)$ & $0.111(4)$ \\
Zr1 & $0.25005(2)$ & $0.25005(2)$ & $0.25005(2)$ & $0.00552(4)$ & \\
O1 & $0.19586(18)$ & $0.28163(18)$ & $0.10116(16)$ & $0.0118(3)$ & \\
O2 & $0.46910(17)$ & $0.55410(17)$ & $0.65064(16)$ & $0.0114(2)$ & \\
Li1 & $0.428(3)$ & $0.593(2)$ & $0.813(2)$ & $0.029(5)$ & $0.33333(14)$ \\
Li2 & $0.642(3)$ & $0.178(2)$ & $0.065(2)$ & $0.029(5)$ & $0.33333(14)$ \\
Ga2 & 0.3750 & 0.5000 & 0.7500 & $0.0061(4)$ & $0.0857(12)$ \\
Li22 & 0.3750 & 0.5000 & 0.7500 & $0.0061(4)$ & $0.743(4)$ \\
Ga3 & 0.2500 & 0.3750 & 0.0000 & $0.0110(9)$ & $0.0403(12)$ \\
Li32 & 0.2500 & 0.3750 & 0.0000 & $0.0110(9)$ & $0.879(4)$ \\
\hline
\end{tabular}

Atomic displacement parameters $\left(\AA^{2}\right)$

\begin{tabular}{lllllll}
\hline & $U^{11}$ & $U^{22}$ & $U^{33}$ & $U^{12}$ & $U^{13}$ & $U^{23}$ \\
\hline La1 & $0.00912(4)$ & $0.00656(5)$ & $0.00668(5)$ & 0.000 & 0.000 & $-0.00141(3)$ \\
Ga1 & $0.00912(4)$ & $0.00656(5)$ & $0.00668(5)$ & 0.000 & 0.000 & $-0.00141(3)$ \\
Zr1 & $0.00552(4)$ & $0.00552(4)$ & $0.00552(4)$ & $0.00021(3)$ & $0.00021(3)$ & $0.00021(3)$ \\
O1 & $0.0129(6)$ & $0.0138(6)$ & $0.0087(5)$ & $0.0014(5)$ & $-0.0007(5)$ & $0.0006(5)$ \\
O2 & $0.0131(6)$ & $0.0116(6)$ & $0.0096(5)$ & $0.0010(5)$ & $0.0000(5)$ & $-0.0013(4)$ \\
Li1 & $0.051(15)$ & $0.015(6)$ & $0.022(6)$ & $-0.013(9)$ & $-0.013(8)$ & $0.006(6)$ \\
Li2 & $0.051(15)$ & $0.015(6)$ & $0.022(6)$ & $-0.013(9)$ & $-0.013(8)$ & $0.006(6)$ \\
Ga2 & $0.0078(10)$ & $0.0052(6)$ & $0.0052(6)$ & 0.000 & 0.000 & 0.000 \\
Li22 & $0.0078(10)$ & $0.0052(6)$ & $0.0052(6)$ & 0.000 & 0.000 & 0.000 \\
Ga3 & $0.0100(14)$ & $0.013(2)$ & $0.0100(14)$ & 0.000 & 0.000 & 0.000 \\
Li32 & $0.0100(14)$ & $0.013(2)$ & $0.0100(14)$ & 0.000 & 0.000 & 0.000 \\
\hline
\end{tabular}

Geometric parameters $\left(\AA,{ }^{\circ}\right)$

\begin{tabular}{|c|c|c|c|}
\hline $\mathrm{La} 1-\mathrm{O} 1^{\mathrm{i}}$ & $2.496(2)$ & $\mathrm{O} 2-\mathrm{Li}^{2 x \mathrm{xii}}$ & $2.09(3)$ \\
\hline $\mathrm{La} 1-\mathrm{O} 1^{\mathrm{ii}}$ & $2.496(2)$ & $\mathrm{O} 2-\mathrm{Zr} 1^{\text {xxiv }}$ & $2.113(2)$ \\
\hline $\mathrm{La} 1-\mathrm{O} 2^{\mathrm{iii}}$ & $2.520(2)$ & $\mathrm{O} 2-\mathrm{Li} 1$ & $2.23(4)$ \\
\hline $\mathrm{La} 1-\mathrm{O} 2^{\mathrm{iv}}$ & $2.520(2)$ & $\mathrm{O} 2-\mathrm{La} 1^{\mathrm{xxv}}$ & $2.520(2)$ \\
\hline $\mathrm{La} 1-\mathrm{O}^{2}{ }^{\mathrm{v}}$ & $2.590(2)$ & $\mathrm{O} 2-\mathrm{Li} 2^{\mathrm{xxvi}}$ & $2.54(3)$ \\
\hline $\mathrm{La} 1-\mathrm{O} 2^{\mathrm{vi}}$ & $2.590(2)$ & $\mathrm{O} 2-\mathrm{La} 1^{\mathrm{xxiii}}$ & $2.590(2)$ \\
\hline $\mathrm{La} 1-\mathrm{O} 1^{\mathrm{vii}}$ & 2.595 (2) & $\mathrm{O} 2-\mathrm{Li} 2^{\mathrm{xxvii}}$ & $2.61(4)$ \\
\hline $\mathrm{La} 1-\mathrm{O} 1^{\text {viii }}$ & $2.595(2)$ & $\mathrm{Li} 1-\mathrm{O} 2^{\mathrm{xxviii}}$ & $1.91(4)$ \\
\hline $\mathrm{Zr} 1-\mathrm{O} 1^{\mathrm{ix}}$ & $2.095(2)$ & $\mathrm{Li} 1-\mathrm{O} 2^{\mathrm{xxii}}$ & $2.04(3)$ \\
\hline
\end{tabular}




\begin{tabular}{|c|c|}
\hline $\mathrm{Zr} 1-\mathrm{O}^{\mathrm{x}}$ & $2.095(2)$ \\
\hline $\mathrm{Zr} 1-\mathrm{O} 1$ & $2.095(2)$ \\
\hline $\mathrm{Zr} 1-\mathrm{O} 2^{\mathrm{xi}}$ & $2.113(2)$ \\
\hline $\mathrm{Zr} 1-\mathrm{O} 2^{\mathrm{xii}}$ & $2.113(2)$ \\
\hline $\mathrm{Zr} 1-\mathrm{O} 2^{\mathrm{xiii}}$ & $2.113(2)$ \\
\hline $\mathrm{O} 1-\mathrm{Li} 2^{\mathrm{xiv}}$ & $1.90(3)$ \\
\hline $\mathrm{O} 1-\mathrm{Ga} 3$ & $1.918(2)$ \\
\hline $\mathrm{O} 1-\mathrm{Li}^{\mathrm{xv}}$ & $2.16(3)$ \\
\hline $\mathrm{O} 1-\mathrm{Li}^{\mathrm{xvi}}$ & $2.22(4)$ \\
\hline $\mathrm{O} 1-\mathrm{Li}^{\mathrm{xvii}}$ & $2.32(3)$ \\
\hline $\mathrm{O} 1-\mathrm{La} 1^{\mathrm{xviii}}$ & $2.496(2)$ \\
\hline $\mathrm{O} 1-\mathrm{La} 1^{\mathrm{xix}}$ & $2.595(2)$ \\
\hline O1-Li1 ${ }^{\mathrm{xx}}$ & $2.65(3)$ \\
\hline $\mathrm{O} 2-\mathrm{Ga} 2$ & $1.908(2)$ \\
\hline $\mathrm{O} 2-\mathrm{Li}^{\mathrm{xxi}}$ & $1.91(4)$ \\
\hline $\mathrm{O} 2-\mathrm{Li}^{\mathrm{xxii}}$ & $2.04(3)$ \\
\hline 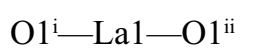 & 73.18 (10) \\
\hline $\mathrm{O} 1^{\mathrm{i}}-\mathrm{La} 1-\mathrm{O} 2^{\mathrm{iii}}$ & $160.55(5)$ \\
\hline 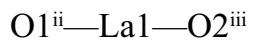 & $111.27(5)$ \\
\hline $\mathrm{O} 1^{\mathrm{i}}-\mathrm{La} 1-\mathrm{O} 2^{\mathrm{iv}}$ & $111.27(5)$ \\
\hline 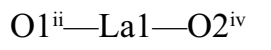 & $160.55(5)$ \\
\hline 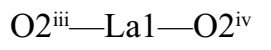 & $71.21(10)$ \\
\hline $\mathrm{O} 1^{\mathrm{i}-\mathrm{La} 1-\mathrm{O} 2^{\mathrm{v}}}$ & 73.18 (7) \\
\hline 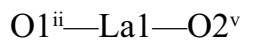 & $95.73(8)$ \\
\hline $\mathrm{O} 2^{\mathrm{iii}-\mathrm{La} 1-\mathrm{O} 2^{\mathrm{v}}}$ & $123.67(5)$ \\
\hline $\mathrm{O} 2^{\mathrm{iv}}-\mathrm{La} 1-\mathrm{O} 2^{\mathrm{v}}$ & $68.75(9)$ \\
\hline $\mathrm{O} 1^{\mathrm{i}}-\mathrm{La} 1-\mathrm{O} 2^{\mathrm{vi}}$ & $95.73(8)$ \\
\hline $\mathrm{O} 1^{\mathrm{ii}}-\mathrm{La} 1-\mathrm{O} 2^{\mathrm{vi}}$ & $73.18(7)$ \\
\hline 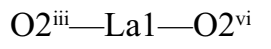 & $68.75(9)$ \\
\hline $\mathrm{O} 2^{\mathrm{iv}}-\mathrm{La} 1-\mathrm{O} 2^{\mathrm{vi}}$ & $123.67(5)$ \\
\hline $\mathrm{O} 2^{\mathrm{v}}-\mathrm{La} 1-\mathrm{O} 2^{\mathrm{vi}}$ & $166.44(9)$ \\
\hline 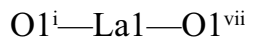 & $125.03(5)$ \\
\hline 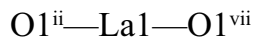 & $68.53(9)$ \\
\hline 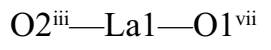 & $72.72(6)$ \\
\hline $\mathrm{O} 2^{\mathrm{iv}}-\mathrm{La} 1-\mathrm{O} 1^{\mathrm{vii}}$ & $94.96(7)$ \\
\hline $\mathrm{O} 2^{\mathrm{v}}-\mathrm{La} 1-\mathrm{O} 1^{\mathrm{vii}}$ & $73.07(5)$ \\
\hline 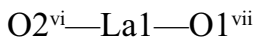 & $108.77(5)$ \\
\hline 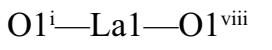 & $68.53(9)$ \\
\hline 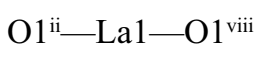 & $125.03(5)$ \\
\hline 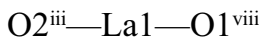 & $94.96(7)$ \\
\hline $\mathrm{O} 2^{\mathrm{iv}}-\mathrm{La} 1-\mathrm{O} 1^{\text {viii }}$ & $72.72(6)$ \\
\hline $\mathrm{O} 2^{\mathrm{v}}-\mathrm{La} 1-\mathrm{O} 1^{\mathrm{viii}}$ & $108.77(5)$ \\
\hline 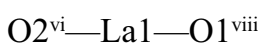 & $73.07(5)$ \\
\hline 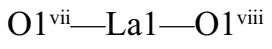 & $165.12(9)$ \\
\hline $\mathrm{O} 1^{\mathrm{ix}}-\mathrm{Zr} 1-\mathrm{O} 1^{\mathrm{x}}$ & $86.37(10)$ \\
\hline 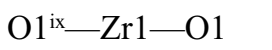 & $86.37(10)$ \\
\hline $\mathrm{O} 1^{\mathrm{x}}-\mathrm{Zr} 1-\mathrm{O} 1$ & $86.37(10)$ \\
\hline
\end{tabular}

\begin{tabular}{|c|c|}
\hline Li1 $-O 1^{\text {xxix }}$ & $2.16(3)$ \\
\hline $\mathrm{Li} 1-\mathrm{O} 1^{\mathrm{xxx}}$ & $2.65(3)$ \\
\hline $\mathrm{Li} 2-\mathrm{O} 1^{\text {viii }}$ & $1.90(3)$ \\
\hline $\mathrm{Li} 2-\mathrm{O} 2^{\mathrm{vi}}$ & $2.09(3)$ \\
\hline $\mathrm{Li} 2-\mathrm{O} 1^{\mathrm{xvi}}$ & $2.22(4)$ \\
\hline $\mathrm{Li} 2-\mathrm{O} 1^{\mathrm{xxxi}}$ & $2.32(3)$ \\
\hline $\mathrm{Li} 2-\mathrm{O} 2^{\mathrm{xxxii}}$ & $2.54(3)$ \\
\hline $\mathrm{Li} 2-\mathrm{O} 2^{\mathrm{xxxiii}}$ & $2.61(4)$ \\
\hline Li2- Li2 $^{\text {xxiv }}$ & $2.68(6)$ \\
\hline $\mathrm{Ga} 2-\mathrm{O} 2^{\mathrm{xxi}}$ & $1.908(2)$ \\
\hline $\mathrm{Ga} 2-\mathrm{O} 2^{\mathrm{xxii}}$ & $1.908(2)$ \\
\hline $\mathrm{Ga} 2-\mathrm{O} 2^{\mathrm{xxviii}}$ & $1.908(2)$ \\
\hline $\mathrm{Ga3}-\mathrm{O} 1^{\mathrm{xxx}}$ & $1.918(2)$ \\
\hline $\mathrm{Ga} 3-\mathrm{O} 1^{\mathrm{xxxvi}}$ & $1.918(2)$ \\
\hline Ga3-O1 & 1.918 \\
\hline
\end{tabular}

La $1^{\text {xviii }-O} 1-\mathrm{Li}^{\mathrm{xx}}$

$79.5(6)$

La ${ }^{x i x}-O 1-$ Li $^{1 \times x}$

$68.9(8)$

$\mathrm{Ga} 2-\mathrm{O} 2-\mathrm{Li} 1^{\mathrm{xxi}}$

$\mathrm{Ga} 2-\mathrm{O} 2-\mathrm{Li} 1^{\text {xxii }}$

$\mathrm{Li}^{\mathrm{xxi}}-\mathrm{O} 2-\mathrm{Li}^{\mathrm{xxii}}$

$\mathrm{Ga} 2-\mathrm{O} 2-\mathrm{Li}^{\text {xxiii }}$

$\mathrm{Li} 1^{\mathrm{xxi}}-\mathrm{O} 2-\mathrm{Li} 2^{\text {xxiii }}$

Li1 ${ }^{\mathrm{xxi}}-\mathrm{O} 2-\mathrm{Li} 2^{\mathrm{xxii}}$

$\mathrm{Ga} 2-\mathrm{O} 2-\mathrm{Zr}^{\text {xxiv }}$

$\mathrm{Li}^{\mathrm{xxi}}-\mathrm{O} 2-\mathrm{Zr} 1^{\text {xxiv }}$

Li1 ${ }^{\text {xxii }}-\mathrm{O} 2-\mathrm{Zr} 1^{\text {xiv }}$

$\mathrm{Li} 2^{\text {xxiii }}-\mathrm{O} 2-\mathrm{Zr}^{\text {xxiv }}$

$\mathrm{Ga} 2-\mathrm{O} 2-\mathrm{Li} 1$

$\mathrm{Li}^{\mathrm{xxi}}-\mathrm{O} 2-\mathrm{Li} 1$

$\mathrm{Li} 1^{\text {xii }}-\mathrm{O} 2-\mathrm{Li} 1$

Li2 ${ }^{\text {xxii }}-\mathrm{O} 2-\mathrm{Li} 1$

$\mathrm{Zr} 1^{\text {xiv }}-\mathrm{O} 2-\mathrm{Li} 1$

$\mathrm{Ga} 2-\mathrm{O} 2-\mathrm{La}^{\mathrm{xxv}}$

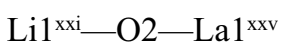

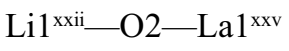

$\mathrm{Li} 2^{\mathrm{xxiii}}-\mathrm{O} 2-\mathrm{La}^{\mathrm{xxv}}$

$\mathrm{Zr}^{1 \mathrm{xiv}}-\mathrm{O} 2-\mathrm{La}^{\mathrm{xxv}}$

$\mathrm{Li} 1-\mathrm{O} 2-\mathrm{La}^{\mathrm{xxv}}$

$\mathrm{Ga} 2-\mathrm{O} 2-\mathrm{Li}^{\mathrm{xxvi}}$

$\mathrm{Li} 1^{\mathrm{xxi}}-\mathrm{O} 2-\mathrm{Li} 2^{\mathrm{xxvi}}$

$\mathrm{Li} 1^{\text {xxii }}-\mathrm{O} 2-\mathrm{Li}^{\mathrm{xxvi}}$

$\mathrm{Li} 2^{\text {xxiii }}-\mathrm{O} 2-\mathrm{Li}^{\mathrm{xxvi}}$

$\mathrm{Zr} 1^{\text {xxiv }}-\mathrm{O} 2-\mathrm{Li}^{\mathrm{xxvi}}$

$\mathrm{Li} 1-\mathrm{O} 2-\mathrm{Li}^{\mathrm{xxvi}}$

$\mathrm{La} 1^{\mathrm{xxv}}-\mathrm{O} 2-\mathrm{Li} 2^{\mathrm{xxvi}}$

$\mathrm{Ga} 2-\mathrm{O} 2-\mathrm{La} 1^{\text {xxii }}$
$49.8(10)$

$48.0(8)$

$77.4(11)$

$66.9(11)$

$18.2(10)$

85.1 (16)

128.57 (12)

104.4 (9)

87.4 (8)

88.5 (10)

44.9 (8)

72.9 (12)

85.9 (12)

89.7 (13)

$173.2(7)$

$94.15(8)$

$143.6(9)$

80.5 (11)

$161.0(11)$

103.05 (9)

77.0 (9)

$57.6(8)$

76.3 (11)

99.9 (14)

$91.0(11)$

$172.6(9)$

$14.0(8)$

79.4 (7)

$122.63(10)$ 


\begin{tabular}{|c|c|}
\hline $\mathrm{O} 1^{\mathrm{ix}}-\mathrm{Zr} 1-\mathrm{O} 2^{\mathrm{xi}}$ & $93.51(9)$ \\
\hline $\mathrm{O} 1^{\mathrm{x}}-\mathrm{Zr} 1-\mathrm{O} 2^{\mathrm{xi}}$ & $179.59(11)$ \\
\hline $\mathrm{O} 1-\mathrm{Zr} 1-\mathrm{O}^{\mathrm{xi}}$ & $94.02(9)$ \\
\hline $\mathrm{O} 1^{\mathrm{ix}}-\mathrm{Zr} 1-\mathrm{O} 2^{\mathrm{xii}}$ & $94.02(9)$ \\
\hline $\mathrm{O} 1^{\mathrm{x}}-\mathrm{Zr} 1-\mathrm{O} 2^{\mathrm{xii}}$ & $93.51(9)$ \\
\hline $\mathrm{O} 1-\mathrm{Zr} 1-\mathrm{O} 2^{\mathrm{xii}}$ & $179.59(11)$ \\
\hline $\mathrm{O} 2^{\mathrm{xi}}-\mathrm{Zr} 1-\mathrm{O} 2^{\mathrm{xii}}$ & $86.10(9)$ \\
\hline $\mathrm{O} 1^{\mathrm{ix}}-\mathrm{Zr} 1-\mathrm{O} 2^{\mathrm{xiii}}$ & $179.59(11)$ \\
\hline $\mathrm{O}^{2}-\mathrm{Zr} 1-\mathrm{O}^{\mathrm{x}} \mathrm{xii}$ & $94.02(9)$ \\
\hline $\mathrm{O} 1-\mathrm{Zr} 1-\mathrm{O} 2^{\mathrm{xiii}}$ & $93.51(9)$ \\
\hline $\mathrm{O} 2^{\mathrm{xi}}-\mathrm{Zr} 1-\mathrm{O} 2^{\mathrm{xiii}}$ & $86.10(9)$ \\
\hline $\mathrm{O} 2^{\mathrm{xii}}-\mathrm{Zr} 1-\mathrm{O} 2^{\mathrm{xiii}}$ & $86.10(9)$ \\
\hline $\mathrm{Li} 2^{\mathrm{xiv}}-\mathrm{O} 1-\mathrm{Ga} 3$ & $55.1(12)$ \\
\hline $\mathrm{Li} 2^{\mathrm{xiv}}-\mathrm{O} 1-\mathrm{Zr} 1$ & $100.2(12)$ \\
\hline $\mathrm{Ga} 3-\mathrm{O} 1-\mathrm{Zr} 1$ & $129.12(12)$ \\
\hline $\mathrm{Li}^{\mathrm{xiv}}-\mathrm{O} 1-\mathrm{Li}^{\mathrm{xv}}$ & $17.3(11)$ \\
\hline $\mathrm{Ga} 3-\mathrm{O} 1-\mathrm{Li}^{\mathrm{xv}}$ & $71.3(8)$ \\
\hline $\mathrm{Zr} 1-\mathrm{O} 1-\mathrm{Li}^{\mathrm{xv}}$ & $84.8(8)$ \\
\hline $\mathrm{Li} 2^{\mathrm{xiv}}-\mathrm{O} 1-\mathrm{Li} 2^{\mathrm{xvi}}$ & $80.8(13)$ \\
\hline $\mathrm{Ga} 3-\mathrm{O} 1-\mathrm{Li}^{2 \mathrm{xi}}$ & $50.0(9)$ \\
\hline $\mathrm{Zr} 1-\mathrm{O} 1-\mathrm{Li} 2^{\mathrm{xvi}}$ & $85.8(9)$ \\
\hline $\mathrm{Li} 1^{\mathrm{xv}}-\mathrm{O} 1-\mathrm{Li} 2^{\mathrm{xvi}}$ & $87.3(14)$ \\
\hline $\mathrm{Li} 2^{\mathrm{xiv}}-\mathrm{O} 1-\mathrm{Li} 2^{\mathrm{xvii}}$ & $78.1(15)$ \\
\hline $\mathrm{Ga} 3-\mathrm{O} 1-\mathrm{Li}^{\mathrm{xvii}}$ & $48.2(10)$ \\
\hline $\mathrm{Zr} 1-\mathrm{O} 1-\mathrm{Li} 2^{\mathrm{xvii}}$ & $177.3(10)$ \\
\hline $\mathrm{Li} 1^{\mathrm{xv}}-\mathrm{O} 1-\mathrm{Li} 2^{\mathrm{xvii}}$ & $93.8(10)$ \\
\hline $\mathrm{Li} 2^{\mathrm{xvi}}-\mathrm{O} 1-\mathrm{Li} 2^{\mathrm{xvii}}$ & $91.8(16)$ \\
\hline $\mathrm{Li} 2^{\mathrm{xiv}}-\mathrm{O} 1-\mathrm{La} 1^{\mathrm{xviii}}$ & $147.5(13)$ \\
\hline Ga3-O1-La1 ${ }^{\text {xviii }}$ & $92.55(8)$ \\
\hline $\mathrm{Zr} 1-\mathrm{O} 1-\mathrm{La} 1^{\mathrm{xviii}}$ & $103.48(9)$ \\
\hline $\mathrm{Li} 1^{\mathrm{xv}}-\mathrm{O} 1-\mathrm{La} 1^{\mathrm{xviii}}$ & $163.4(9)$ \\
\hline $\mathrm{Li} 2^{\mathrm{xvi}}-\mathrm{O} 1-\mathrm{La}^{\mathrm{xviii}}$ & $79.1(7)$ \\
\hline $\mathrm{Li} 2^{\mathrm{xvii}}-\mathrm{O} 1-\mathrm{La} 1^{\mathrm{xviii}}$ & $77.3(7)$ \\
\hline $\mathrm{Li} 2^{\mathrm{xiv}}-\mathrm{O} 1-\mathrm{La} 1^{\mathrm{xix}}$ & $94.7(9)$ \\
\hline $\mathrm{Ga} 3-\mathrm{O} 1-\mathrm{La}^{\mathrm{xix}}$ & $123.13(10)$ \\
\hline $\mathrm{Zr} 1-\mathrm{O} 1-\mathrm{La} 1^{\mathrm{xix}}$ & $100.26(9)$ \\
\hline $\mathrm{Li} 1^{\mathrm{xv}}-\mathrm{O} 1-\mathrm{La}^{\mathrm{xix}}$ & $89.9(10)$ \\
\hline $\mathrm{Li} 2^{\mathrm{xvi}}-\mathrm{O} 1-\mathrm{La} 1^{\mathrm{xix}}$ & $173.1(9)$ \\
\hline $\mathrm{Li} 2^{\mathrm{xvii}}-\mathrm{O} 1-\mathrm{La}^{\mathrm{xix}}$ & $82.1(10)$ \\
\hline La $1^{\text {xviii }}-\mathrm{O} 1-\mathrm{La} 1^{\mathrm{xix}}$ & $102.50(8)$ \\
\hline $\mathrm{Li} 2^{\mathrm{xiv}}-\mathrm{O} 1-\mathrm{Li} 1^{\mathrm{xx}}$ & $81.4(12)$ \\
\hline $\mathrm{Ga} 3-\mathrm{O} 1-\mathrm{Li} 1^{\mathrm{xx}}$ & $60.5(8)$ \\
\hline $\mathrm{Zr} 1-\mathrm{O} 1-\mathrm{Li1}^{\mathrm{xx}}$ & $169.2(8)$ \\
\hline $\mathrm{Li} 1^{\mathrm{xv}}-\mathrm{O} 1-\mathrm{Li} 1^{\mathrm{xx}}$ & $95.1(9)$ \\
\hline
\end{tabular}

\begin{tabular}{|c|c|}
\hline Li1 $1^{x x i}-O 2-L a 1^{x x i i i}$ & $95.8(10)$ \\
\hline $\mathrm{Li} 1^{\mathrm{xxii}}-\mathrm{O} 2-\mathrm{La} 1^{\mathrm{xxiii}}$ & $170.6(8)$ \\
\hline $\mathrm{Li} 2^{\mathrm{xxiii}}-\mathrm{O} 2-\mathrm{La} 1^{\mathrm{xxiii}}$ & $90.4(8)$ \\
\hline $\mathrm{Zr} 1^{\mathrm{xxiv}}-\mathrm{O} 2-\mathrm{La} 1^{\mathrm{xxiii}}$ & $100.77(8)$ \\
\hline Li1-O2-La1 $1^{\text {xxiii }}$ & $85.8(7)$ \\
\hline $\mathrm{La} 1^{\mathrm{xxy}}-\mathrm{O} 2-\mathrm{La} 1^{\mathrm{xxiii}}$ & $101.98(8)$ \\
\hline $\mathrm{Li} 2^{\mathrm{xxvi}}-\mathrm{O} 2-\mathrm{La} 1^{\mathrm{xxiii}}$ & $71.8(9)$ \\
\hline $\mathrm{Ga} 2-\mathrm{O} 2-\mathrm{Li} 2^{\mathrm{xxvii}}$ & $56.1(7)$ \\
\hline $\mathrm{Li} 1^{\mathrm{xxi}}-\mathrm{O} 2-\mathrm{Li} 2^{\mathrm{xxvii}}$ & $83.4(14)$ \\
\hline $\mathrm{Li} 1^{\mathrm{xxii}}-\mathrm{O} 2-\mathrm{Li} 2^{\mathrm{xxvii}}$ & $8.2(9)$ \\
\hline $\mathrm{Li} 2^{\mathrm{xxiii}}-\mathrm{O} 2-\mathrm{Li} 2^{\mathrm{xxvii}}$ & $89.2(9)$ \\
\hline $\mathrm{Zr} 1^{\mathrm{xxiv}}-\mathrm{O} 2-\mathrm{Li} 2^{\mathrm{xxvii}}$ & $80.4(7)$ \\
\hline Li1-O2-Li2 ${ }^{x x v i i}$ & $93.0(11)$ \\
\hline $\mathrm{La} 1^{\mathrm{xxy}}-\mathrm{O} 2-\mathrm{Li} 2^{\mathrm{xxvii}}$ & $78.2(6)$ \\
\hline $\mathrm{Li} 2^{\mathrm{xxvi}}-\mathrm{O} 2-\mathrm{Li} 2^{\mathrm{xxvii}}$ & $107.0(12)$ \\
\hline $\mathrm{La} 1^{\mathrm{xxiii}}-\mathrm{O} 2-\mathrm{Li} 2^{\mathrm{xxvii}}$ & $178.8(7)$ \\
\hline $\mathrm{O} 2^{\mathrm{xxviii}}-\mathrm{Li} 1-\mathrm{O} 2^{\mathrm{xxii}}$ & $108.5(14)$ \\
\hline $\mathrm{O} 2^{\mathrm{xxviii}}-\mathrm{Li} 1-\mathrm{O} 1^{\mathrm{xxix}}$ & $98.6(18)$ \\
\hline $\mathrm{O} 2^{\mathrm{xxii}}-\mathrm{Li} 1-\mathrm{O} 1^{\mathrm{xxix}}$ & $93.9(12)$ \\
\hline $\mathrm{O} 2^{\mathrm{xxviii}}-\mathrm{Li} 1-\mathrm{O} 2$ & $101.2(12)$ \\
\hline $\mathrm{O} 2^{\mathrm{xxii}}-\mathrm{Li} 1-\mathrm{O} 2$ & $86.8(14)$ \\
\hline $\mathrm{O} 1^{\mathrm{xxix}}-\mathrm{Li} 1-\mathrm{O} 2$ & $158.9(18)$ \\
\hline $\mathrm{O} 2^{\mathrm{xxviii}}-\mathrm{Li} 1-\mathrm{O} 1^{\mathrm{xxx}}$ & $144.7(15)$ \\
\hline $\mathrm{O} 2^{\mathrm{xxii}}-\mathrm{Li} 1-\mathrm{O} 1^{\mathrm{xxx}}$ & $106.5(15)$ \\
\hline $\mathrm{O} 1^{\mathrm{xxix}}-\mathrm{Li} 1-\mathrm{O} 1^{\mathrm{xxx}}$ & $83.1(9)$ \\
\hline $\mathrm{O} 2-\mathrm{Li} 1-\mathrm{O} 1^{\mathrm{xxx}}$ & $76.4(12)$ \\
\hline $\mathrm{O} 1^{\mathrm{vii}-\mathrm{Li} 2-\mathrm{O} 2^{\mathrm{vi}}}$ & $101.0(12)$ \\
\hline $\mathrm{O} 1^{\mathrm{vii}}-\mathrm{Li} 2-\mathrm{O} 1^{\mathrm{xvi}}$ & $102.1(17)$ \\
\hline 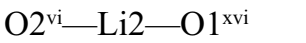 & $91.1(12)$ \\
\hline 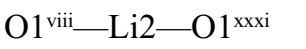 & $98.5(14)$ \\
\hline $\mathrm{O} 2^{\mathrm{vi}}-\mathrm{Li} 2-\mathrm{O} 1^{\mathrm{xxxi}}$ & $160.3(14)$ \\
\hline $\mathrm{O} 1^{\mathrm{xvi}}-\mathrm{Li} 2-\mathrm{O} 1^{\mathrm{xxxi}}$ & $82.0(13)$ \\
\hline $\mathrm{O} 1^{\mathrm{viii}}-\mathrm{Li} 2-\mathrm{O} 2^{\mathrm{xxxii}}$ & $151.8(19)$ \\
\hline 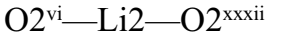 & $86.9(13)$ \\
\hline $\mathrm{O} 1^{\mathrm{xvi}}-\mathrm{Li} 2-\mathrm{O} 2^{\mathrm{xxxii}}$ & $104.8(10)$ \\
\hline 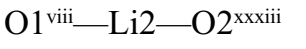 & $84.1(12)$ \\
\hline 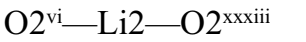 & $85.2(14)$ \\
\hline $\mathrm{O} 1^{\mathrm{xvi}}-\mathrm{Li} 2-\mathrm{O} 2^{\mathrm{xxxiii}}$ & $173.3(16)$ \\
\hline $\mathrm{O} 1^{\mathrm{xxx}}-\mathrm{Li} 2-\mathrm{O} 2^{\mathrm{xxxiii}}$ & $99.7(11)$ \\
\hline $\mathrm{O} 2^{\mathrm{xxxii}}-\mathrm{Li} 2-\mathrm{O} 2^{\mathrm{xxxiii}}$ & $69.5(10)$ \\
\hline $\mathrm{O} 2^{\mathrm{xxi}}-\mathrm{Ga} 2-\mathrm{O} 2^{\mathrm{xxii}}$ & $114.14(7)$ \\
\hline $\mathrm{O} 2^{\mathrm{xxi}}-\mathrm{Ga} 2-\mathrm{O} 2^{\mathrm{xxviii}}$ & $100.49(13)$ \\
\hline $\mathrm{O} 2^{\mathrm{xxii}}-\mathrm{Ga} 2-\mathrm{O} 2^{\mathrm{xxviii}}$ & $114.14(7)$ \\
\hline $\mathrm{O} 1-\mathrm{Ga} 3-\mathrm{O} 1^{\mathrm{xxxv}}$ & $113.48(7)$ \\
\hline
\end{tabular}




\section{supporting information}

$\begin{array}{llll}\mathrm{Li} 2^{\mathrm{xvi}}-\mathrm{O} 1-\mathrm{Li} 1^{\mathrm{xx}} & 105.0(14) & \mathrm{O} 1-\mathrm{Ga} 3-\mathrm{O} 1^{\mathrm{xxxvi}} & 101.73(14) \\ \mathrm{Li}^{\mathrm{xvii}}-\mathrm{O} 1-\mathrm{Li}^{\mathrm{xx}} & 13.3(11) & \mathrm{O} 1^{\mathrm{xxv}}-\mathrm{Ga} 3-\mathrm{O} 1^{\mathrm{xxxi}} & 113.48(7)\end{array}$

Symmetry codes: (i) $-y+3 / 4, x-1 / 4,-z+1 / 4$; (ii) $-y+3 / 4,-x+1 / 4, z+1 / 4$; (iii) $-x+5 / 4,-z+3 / 4, y-1 / 4$; (iv) $-x+5 / 4, z-3 / 4,-y+3 / 4$; (v) $-z+5 / 4, y-3 / 4,-x+3 / 4$; (vi) $-z+5 / 4,-y+3 / 4, x-1 / 4$; (vii) $-z+3 / 4,-y+1 / 4, x+1 / 4$; (viii) $-z+3 / 4, y-1 / 4,-x+1 / 4$; (ix) $y, z, x$; (x) $z, x, y$; (xi) $z-1 / 4, y-1 / 4, x-1 / 4$; (xii) $y-1 / 4, x-1 / 4$, $z-1 / 4$; (xiii) $x-1 / 4, z-1 / 4, y-1 / 4$; (xiv) $-z+1 / 4, y+1 / 4,-x+3 / 4$; (xv) $x-1 / 4,-z+5 / 4,-y+3 / 4$; (xvi) $-x+1,-y+1 / 2, z$; (xvii) $x-1 / 2,-y+1 / 2,-z$; (xviii) $-y+1 / 4$, $-x+3 / 4, z-1 / 4$; (xix) $z-1 / 4,-y+1 / 4,-x+3 / 4$; (xx) $y-1 / 2, z-1 / 2, x-1 / 2$; (xxi) $-x+3 / 4, z-1 / 4,-y+5 / 4$; (xxii) $x,-y+1,-z+3 / 2$; (xxiii) $z+1 / 4,-y+3 / 4,-x+5 / 4$; (xxiv) $y+1 / 4, x+1 / 4, z+1 / 4$; (xxv) $-x+5 / 4, z+1 / 4,-y+3 / 4$; (xxvi) $-z+1 / 2, x,-y+1$; (xxvii) $-z+1 / 2,-x+1, y+1 / 2$; (xxviii) $-x+3 / 4,-z+5 / 4, y+1 / 4$; (xxix) $x+1 / 4,-z+3 / 4,-y+5 / 4$; (xxx) $z+1 / 2, x+1 / 2, y+1 / 2$; (xxxi) $x+1 / 2,-y+1 / 2,-z$; (xxxii) $y,-z+1,-x+1 / 2$; (xxxiii) $-y+1, z-1 / 2,-x+1 / 2$; (xxxiv) $z+3 / 4,-y+1 / 4$, $-x+3 / 4 ;(\mathrm{xxxv}) z+1 / 4,-y+3 / 4,-x+1 / 4$; (xxxvi) $-x+1 / 2, y,-z$; (xxxvii) $-z+1 / 4,-y+3 / 4, x-1 / 4$. 